\title{
A randomized clinical trial of the effectiveness of mechanical traction for sub-groups of patients with low back pain: study methods and rationale
}

\author{
Julie M Fritz ${ }^{* 1,2}$, Anne Thackeray 1 ,2, John D Childs ${ }^{3}$ and Gerard P Brennan
}

\begin{abstract}
Background: Patients with signs of nerve root irritation represent a sub-group of those with low back pain who are at increased risk of persistent symptoms and progression to costly and invasive management strategies including surgery. A period of non-surgical management is recommended for most patients, but there is little evidence to guide non-surgical decision-making. We conducted a preliminary study examining the effectiveness of a treatment protocol of mechanical traction with extension-oriented activities for patients with low back pain and signs of nerve root irritation. The results suggested this approach may be effective, particularly in a more specific sub-group of patients. The aim of this study will be to examine the effectiveness of treatment that includes traction for patients with low back pain and signs of nerve root irritation, and within the pre-defined sub-group.
\end{abstract}

Methods/Design: The study will recruit 120 patients with low back pain and signs of nerve root irritation. Patients will be randomized to receive an extension-oriented treatment approach, with or without the addition of mechanical traction. Randomization will be stratified based on the presence of the pre-defined sub-grouping criteria. All patients will receive 12 physical therapy treatment sessions over 6 weeks. Follow-up assessments will occur after 6 weeks, 6 months, and 1 year. The primary outcome will be disability measured with a modified Oswestry questionnaire. Secondary outcomes will include self-reports of low back and leg pain intensity, quality of life, global rating of improvement, additional healthcare utilization, and work absence. Statistical analysis will be based on intention to treat principles and will use linear mixed model analysis to compare treatment groups, and examine the interaction between treatment and sub-grouping status.

Discussion: This trial will provide a methodologically rigorous evaluation of the effectiveness of using traction for patients with low back pain and signs of nerve root irritation, and will examine the validity of a pre-defined subgrouping hypothesis. The results will provide evidence to inform non-surgical decision-making for these patients.

Trial Registration: This trial has been registered with http://ClinicalTrials.gov: NCT00942227

\section{Background}

\section{The Problem of Low Back Pain with Nerve Root} Involvement

Low back pain is a common condition that is estimated to affect approximately $40 \%$ of the adult population within a 1-month time frame [1]. A majority of cases of LBP are considered "non-specific", with no clearly evident pathoanatomic cause [2]. Among the most common specific causes of LBP are disorders of the lumbar intervertebral

\footnotetext{
*Correspondence: julie.fritz@hsc.utah.edu

1 Rehabilitation Agency, Intermountain Healthcare, Salt Lake City, Utah, USA Full list of author information is available at the end of the article
}

disks resulting in irritation of the lumbar nerve roots. It is estimated that nerve root involvement accompanies approximately $10 \%$ of episodes of LBP [3], with lifetime prevalence estimates ranging from $12 \%$ - 43\% [4,5]. Although LBP accompanied by nerve root involvement accounts for a relatively small percentage of all cases of LBP, the presence of nerve root involvement has been associated with an increased severity of symptoms, heightened risk of chronicity and work absence, and higher health care costs [3,6-8].

Although surgery may offer benefits for some patients with LBP and nerve root involvement [9], non-surgical 
management is recommended as an initial strategy in most cases [10]. However, there appears to be a wide range of non-surgical management strategies undertaken for these patients, with little standardization or evidence to inform decision-making [11]. Many patients with LBP and nerve root involvement receive physical therapy [12], yet there is currently little research examining the potential benefits of physical therapy relative to other non-surgical options. A recent study suggests there may be some benefit to receiving physical therapy compared to management by a general practitioner alone [13], however the benefits of physical therapy were not consistent across all outcomes in the study, and the results did not support the overall cost-effectiveness of physical therapy [14]. Physical therapists use a wide variety of treatments for patients with LBP and nerve root involvement [15], and sub-optimal outcomes may be at least partly attributable to the relatively sparse evidence on the specific treatment options that are most effective for these patients [16,17]. It is also unclear if there is meaningful heterogeneity in the response to different physical therapy treatment options, which would indicate a potential benefit in identifying more homogeneous sub-groups within the larger group of patients with LBP and nerve root involvement.

\section{Mechanical Traction as a Treatment for Low Back Pain with Nerve Root Involvement}

Axial traction has been used for centuries as a treatment for LBP and was popularized by Cyriax as an intervention for patients with nerve root involvement due to lumbar intervertebral disk herniation [18]. Evidence-based guidelines and systematic reviews have generally not supported the use of traction for patients with LBP [19-21]. Despite the lack of research support, surveys indicate continued use of traction, in various forms and delivered by various providers, for patients with LBP [22-25]. The rationale offered by those who advocate the use of traction despite the lack of supporting evidence is the low methodological quality and questionable external validity of the majority of the research examining traction [26]. External validity concerns have centered on divergences between the delivery of traction in research and clinical practice, including questions about dosage parameters (traction force and duration), the use of concomitant interventions, and patient selection [27]. Specifically, traction has generally been researched as a stand-alone treatment, whereas clinicians often deliver traction in conjunction with other treatments, most often exercise interventions [28]. Clinicians also report using traction for selected sub-groups of patients with LBP, whereas research has often included more heterogeneous patient samples. The most common sub-group of patients cited by clinicians as appropriate for traction are those with LBP accompanied by signs of nerve root irritation $[27,28]$, however the accuracy of this clinical perception has not been adequately researched.

\section{Preliminary Research to Identify a Sub-Group of Patients Likely to Benefit from Traction}

As an initial step towards investigating if a more clinically-relevant traction protocol would result in larger treatment effects, and to explore if a specific sub-group of patients with LBP exists for whom evidence would support the use of traction, we conducted a randomized clinical trial [29]. The inclusion criteria for this trial were designed to provide a more homogeneous sample that reflected clinicians' perceptions of the appropriate subgroup of patients most likely to benefit from traction. The trial required patients to have symptoms distal to the buttock accompanied by clinical signs of nerve root irritation. Because we hypothesized that clinically important heterogeneity may exist even within this more narrowlydefined sample of patients, we secondarily examined additional baseline variables, nominated based on expert opinion and observations, for their potential to further define a traction sub-group. The trial randomized patients to one of two treatment groups, both of which received 6 weeks of treatment involving an extension-oriented treatment approach (EOTA) comprised of repeated end-range extension exercises and supplemented with manual therapy and education designed to facilitate centralization of the patient's symptoms. One group also received mechanical traction during the first two weeks of treatment, in addition to this extension-oriented approach (EOTA+traction). The traction protocol was designed to reflect common clinical practice $[28]$ and was informed by expert clinical input [30]. The protocol used a high dosage of traction, with a sustained traction force ranging between $40 \%-60 \%$ of a patient's body weight, delivered for a maximum of 12 minutes during a treatment session. The delivery of traction was combined with concomitant use of the EOTA interventions designed to enhance centralization of symptoms. We provided this high-dose traction during the initial 2 weeks of traction to reflect the clinical perception that traction is most effective when delivered early in the course of treatment, followed by transition to exercise or other patientdirected activities [30].

Analysis of the overall randomized trial results found a significant time $\mathrm{x}$ treatment interaction after 2 weeks, but not at the 6 week follow-up for the outcome of disability (Modified Oswestry questionnaire) (figure 1). The group receiving EOTA+traction experienced greater change after 2 weeks on the Oswestry questionnaire, however the magnitude of the treatment effect was at the margins of clinical significance [31] (mean difference $=7.2$ points (95\% CI: 0.13, 14.3)), and was no longer significant after 6 weeks. Our secondary analysis identified two baseline 


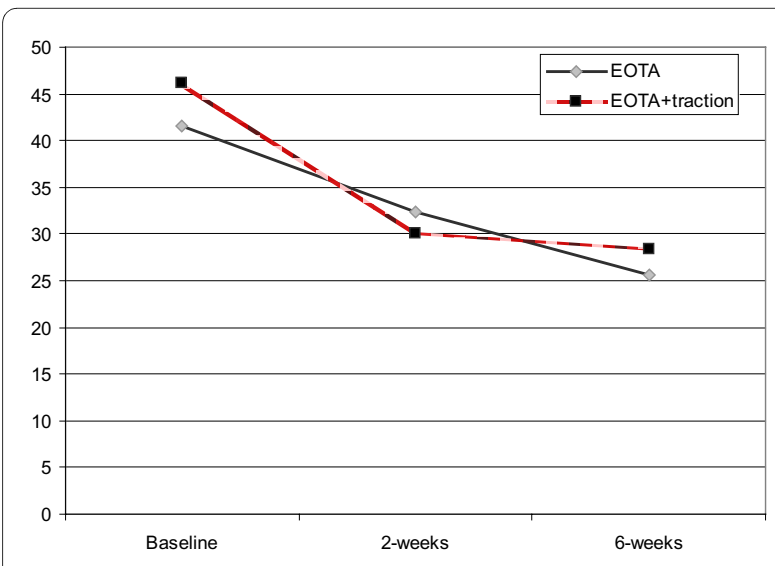

Figure 1 Comparison of patients receiving an Extension-Oriented Treatment Approach (EOTA) with or without traction in the preliminary randomized trial [29]. A significant treatment effect favoring traction was evident after 2 weeks, but not after 6 weeks.

variables that significantly interacted with the treatment provided: the presence of peripheralization of symptoms with extension movement, and a positive crossed straight leg raise test. Analysis of the results using the presence of either of these two factors to preliminarily define a subgroup of likely traction responders (table 1) demonstrated a significant time $\mathrm{x}$ treatment $\mathrm{x}$ sub-group status, 3-way interaction at both the 2- and 6-week follow-up (figure 2). The nature of this interaction indicated that patients who met the preliminary sub-grouping criteria and received traction experienced greater change in disability across time than those who did not meet the subgrouping criteria and received traction, or those who met the criteria but did not receive traction (figure 2). The presence of this interaction suggests the sub-grouping criteria identified in this preliminary study may represent an effect modifier specific to the use of lumbar traction (i.e., the response to lumbar traction is expected to differ based on sub-grouping status) [32]. Additional research in different patient samples is needed to validate or refute this preliminary finding [33].

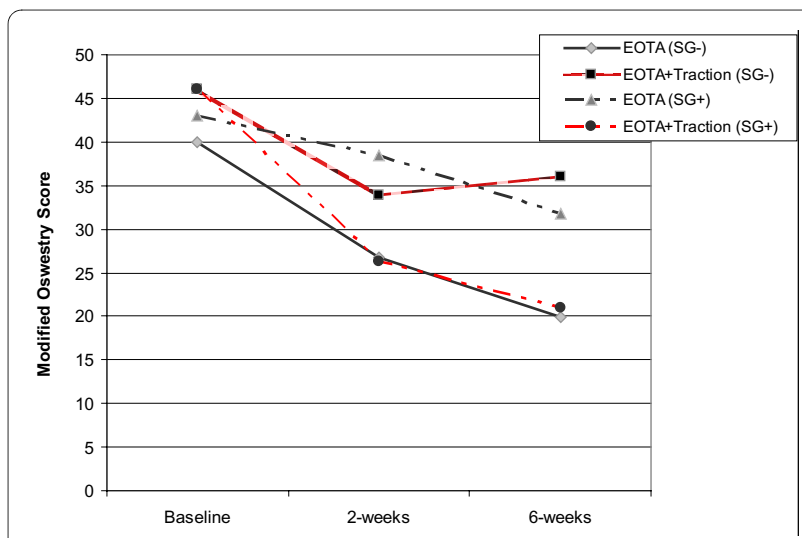

Figure 2 Comparison of patients receiving an Extension-Oriented Treatment Approach (EOTA) with or without traction based on status on the sub-grouping (SG) criteria for traction in the preliminary randomized trial [29]. A significant 3-way interaction between treatment, sub-grouping status, and time was evident after 2 and 6 weeks.

\section{Methods/Design \\ Overview of research design}

The study will be a randomized controlled trial comparing an extension-oriented treatment approach (EOTA) with or without the addition of mechanical traction. The study will include patients with signs and symptoms consistent with lumbar nerve root irritation. Randomization will be stratified based on the preliminary sub-grouping criteria (table 1). The study design and subject flow are outlined in figure 3 . Subjects in each treatment group will receive 12 individual treatment sessions with a physical therapist delivered over a 6-week period. Patients randomized to the traction group will receive traction combined with the EOTA throughout the 6-week treatment period. Outcomes will be assessed at the conclusion of treatment ( 6 weeks), and after 6 months and 1 year. The study has been approved by the Institutional Review Boards at the University of Utah, Intermountain Healthcare, Inc., and Wilford Hall Medical Center.

\section{Table 1: Definition of preliminary criteria to define a sub-group of patients likely to benefit from traction.}

\section{Criterion}

\section{Definition}

1. Peripheralization with extension movement
Presence of peripheralization with at least one of the following extension movements during the baseline examination: single extension standing, repeated extension standing, sustained extension prone (prone on elbows), or repeated extension prone (prone press-ups).

Peripheralization is judged to have occurred if a pain or paresthesia moves distally away from the spine toward the periphery, or paresthesia or a neurological sign is worsened or produced during or after the movement [48]

\section{Positive crossed straight leg raise test \\ Reproduction of familiar symptoms in the symptomatic lower extremity with passive straight leg raising of the unaffected leg at an angle of $70^{\circ}$ or less [49].}




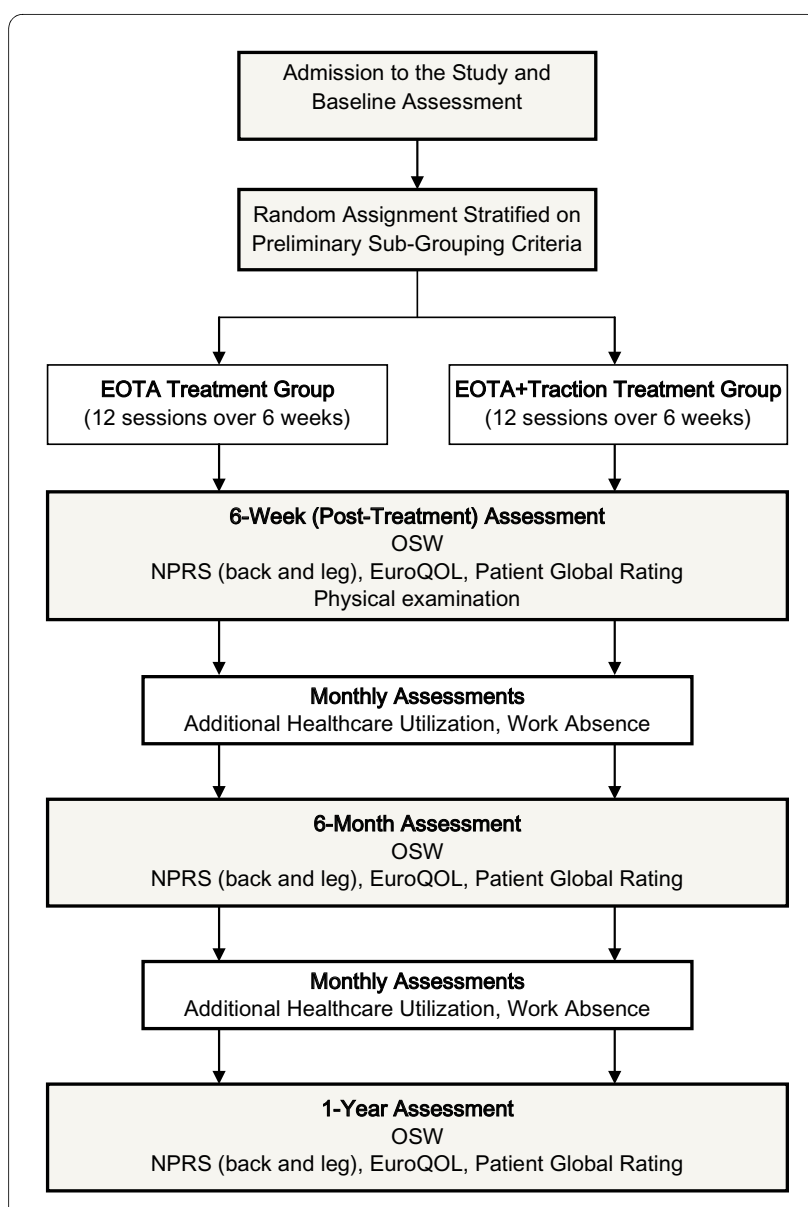

Figure 3 Participant flow through the study

\section{Hypotheses}

1. The treatment group receiving traction in addition to an EOTA intervention will have better outcomes than the treatment group receiving the EOTA intervention without traction after 6 weeks.

2 . The treatment group receiving traction in addition to an EOTA intervention will have better outcomes than the treatment group receiving the EOTA intervention without traction after 6 months and 1 year.

3. Patients who are positive on the preliminary traction sub-grouping criteria and receive traction in addition to an EOTA intervention will have better outcomes at all time points than:

a. patients who are negative on the preliminary traction sub-grouping criteria and receive traction in addition to an EOTA intervention

b. patients who are positive on the preliminary traction sub-grouping criteria and do not receive traction in addition to an EOTA intervention.

\section{Subject Recruitment}

A total of 120 subjects will be recruited for participation. Subjects will be recruited from physician (general prac- tice, physiatry, orthopedic surgery) and physical therapist (outpatient orthopedic) practices in Salt Lake City, Utah and San Antonio, Texas in the United States. Interested individuals from these practices will be screened for eligibility based on the inclusion/exclusion criteria. Subjects must meet each of the study's inclusion criteria, and not have any of the exclusion criteria in order to be eligible for enrollment in this study (table 2). These criteria are designed to recruit a sample of subjects with signs and symptoms of nerve root irritation without contraindications to the study treatments. Subjects over age 60, and those reporting an absence of symptoms when seated, are excluded to reduce to likelihood of including subjects whose LBP is attributable to lumbar spinal stenosis, for whom extension exercises may be contra-indicated. All potential subjects screened for eligibility will be recorded as well as the reason for ineligibility of potential subjects who are not enrolled in the study.

\section{Outcome Measures}

The baseline evaluation of the subject will be conducted following confirmation of eligibility and the provision of informed consent. Demographic characteristics will be collected including the subject's age, gender, employment status, LBP history, prior treatments for LBP, and treatment expectations. Subjects' fear-avoidance beliefs regarding physical activity and work will be assessed using the Fear-Avoidance Belief Questionnaire [34], and pain catastrophizing will be evaluated with the Pain Catastrophizing Scale [35]. Measures of treatment outcome will be collected at the baseline evaluation and repeated after 6 weeks, 6 months, and 1 year.

The primary outcome measure for determining treatment effectiveness will be LBP-related disability measured using a Modified version of the Oswestry disability index (OSW) [31]. The OSW consists of 10 items assessing different aspects of pain and function related to LBP. Each item is scored from 0-5 with higher scores representing greater disability. The OSW is a recommended and widely-used measure of outcome in clinical trials evaluating treatments for patients with LBP [36], with demonstrated responsiveness to change in individuals with LBP, including those with LBP accompanied by leg pain $[37,38]$. Secondary outcome measures will include average low back and leg pain intensity over the past 24 hours measured using numeric rating scales [39]. Subject's will be asked to rate their current level of pain as well as the best and worst levels of pain over the past 24 hours on 11-point scales ranging from 0 "no pain" to 10 "worst imaginable pain". The mean of the three ratings will represent the average pain intensity. Separate ratings will be collected for LBP and leg pain. Additional secondary outcome measures will be the subject's global rating of change from initiation of treatment to present using a 
Table 2: Inclusion and exclusion criteria for participation in the study.

\begin{tabular}{|c|c|}
\hline Inclusion Criteria & Exclusion Criteria \\
\hline $\begin{array}{l}\text { 1. Chief complaint of pain and/or paresthesia in the lumbar spine } \\
\text { with a distribution of symptoms that has extended distal to the } \\
\text { gluteal fold into at least one lower extremity within the past } 24 \text { hours. }\end{array}$ & $\begin{array}{l}\text { 1. Known serious spinal pathology (e.g., spinal tumor, fracture, } \\
\text { infectious disorder, osteoporosis or other bone demineralizing } \\
\text { condition), or suspicion of serious pathology based on red flags } \\
\text { noted in the general medical screening. }\end{array}$ \\
\hline 2. Modified Oswestry score $\geq 20 \%$ & $\begin{array}{l}\text { 2. Evidence of central nervous system involvement, including } \\
\text { symptoms of cauda equina syndrome (i.e. loss of bowel/bladder } \\
\text { control or saddle region paresthesia) or the presence of } \\
\text { pathological reflexes (i.e. positive Babinski) in the physical } \\
\text { examination. }\end{array}$ \\
\hline 3. Age at least 18 and less than 60 years & $\begin{array}{l}\text { 3. Patient report of a the complete absence of LBP and leg } \\
\text { symptoms when seated }\end{array}$ \\
\hline 4. At least one of the following signs of nerve root compression: & $\begin{array}{l}\text { 5. Recent (within the past } 2 \text { weeks) epidural steroid injection for } \\
\text { LBP and/or leg pain }\end{array}$ \\
\hline $\begin{array}{l}\text { I. Positive ipsilateral or contralateral straight leg raise test } \\
\text { (reproduction of leg symptoms with leg raise }<70^{\circ} \text { ) }\end{array}$ & 6. Current pregnancy \\
\hline II. Sensory deficit to pinprick on the ipsilateral lower extremity & $\begin{array}{l}\text { 7. Known inability to comply with the treatment schedule (e.g., } \\
\text { planned vacation, etc.) }\end{array}$ \\
\hline \multicolumn{2}{|l|}{$\begin{array}{l}\text { III. Diminished myotomal strength of the ipsilateral lower } \\
\text { extremity }\end{array}$} \\
\hline $\begin{array}{l}\text { IV. Diminished lower extremity muscle stretch reflex (Quadriceps } \\
\text { or Achilles) of the symptomatic lower extremity }\end{array}$ & \\
\hline
\end{tabular}

15-level scale [40], and health-related quality of life assessed using the Euroqol (EQ-5D) [41].

Subjects' utilization of healthcare resources and associated direct healthcare costs, as well as work absence due to LBP will be recorded during the year following enrollment in the trial. The subject will be contacted by phone or e-mail on a monthly basis following enrollment. At each contact the subject will asked to report any visits to healthcare providers for the management of LBP, any diagnostic (e.g., MRI, radiographs, etc.) or interventional procedures (e.g., injection, surgery, etc.), and any prescription medication purchased for LBP. The subject will also be asked to report the number of days absent from work related to LBP. Direct healthcare costs will be computed by assigning a standardized cost to each healthcare service received based on average procedure cost data from Intermountain Healthcare's health plan.

\section{Randomization}

Assignment of subjects to treatment groups will be performed by a research assistant following completion of all baseline evaluation procedures. Randomization will be stratified based on the presence (or absence) of the preliminary traction sub-grouping criteria. The preliminary subgrouping criteria will be judged positive (SG+) if either of the two sub-grouping variables is identified during the baseline physical examination (table 1). The preliminary sub-grouping criteria will be judged to be absent (SG-) if $\underline{b o t h}$ of the sub-grouping variables are absent during the baseline physical examination. Our preliminary study found 32 out of 64 subjects to be SG+ (50\%), and we anticipate a similar proportion in this study. After completion of all baseline evaluation activities, the research assistant will select and open the next sealed opaque envelope appropriate to the subject's sub-grouping status. Allocation sequences will be generated in permuted block sizes of 6,8 , or 10 by study personnel prior to beginning the trial using a web-based randomization generator http://www.randomization.com. Separate sequences will be generated for each recruitment site.

\section{Interventions}

Subjects will be randomized to one of two treatment groups. One group will receive only the EOTA intervention, the other group will receive the EOTA intervention with the addition of mechanical lumbar traction. Subjects in both treatment groups will receive a total of 12 individual physical therapy treatment sessions delivered over a 6-week treatment period. Three sessions will occur during weeks 1 and 2 of the treatment period, two sessions in weeks 3 and 4, and one session during weeks 5 and 6 .

\section{Extension-Oriented Treatment Approach (EOTA) Group}

The EOTA intervention involves three components. The goals of each component are to promote centralization of symptoms and extension range of motion of the lumbar spine. The first component will be a series of active extension-oriented exercises (table 3). These exercises will consist of sustained and repeated extension movements 
Table 3: Exercise progression within the extension-oriented treatment approach.

\begin{tabular}{|c|c|c|}
\hline Extension-Oriented Exercises & Goal & Options for Progression \\
\hline Prone Lying & 5 minutes & $\begin{array}{l}\text { May use pillow to allow lumbar flexion initially if needed } \\
\text { May shift pelvis laterally if needed } \\
\text { Progress to full lumbar extension without pillows or lateral shift of pelvis } \\
\text { Progress time up to } 5 \text { minutes }\end{array}$ \\
\hline Prone Lying on Elbows & 5 minutes & $\begin{array}{l}\text { May use partial range of extension motion } \\
\text { initially if needed } \\
\text { May shift pelvis laterally if needed } \\
\text { Progress to full lumbar extension with-out lateral } \\
\text { shift of pelvis } \\
\text { Progress time up to } 5 \text { minutes }\end{array}$ \\
\hline Prone Press-Ups & $\begin{array}{l}30 \\
\text { repetitions }\end{array}$ & $\begin{array}{l}\text { May use partial range of extension motion initially if needed } \\
\text { May shift pelvis laterally if needed } \\
\text { Progress to full lumbar extension with-out lateral shift of pelvis } \\
\text { Progress to exhalation during the last repetition to promote increased extension } \\
\text { Progress to therapist over-pressure during repetitions to promote increased extension } \\
\text { Progress up to } 30 \text { repetitions }\end{array}$ \\
\hline Extension in Standing & $\begin{array}{l}30 \\
\text { repetitions }\end{array}$ & $\begin{array}{l}\text { May use partial range of extension motion initially if needed } \\
\text { May shift pelvis laterally if needed } \\
\text { Progress to full lumbar extension with-out lateral shift of pelvis } \\
\text { Progress up to } 30 \text { repetitions }\end{array}$ \\
\hline
\end{tabular}

performed with the subject prone or standing. The exercises may be performed with a lateral component (i.e., shifting of the pelvis in the frontal plane) if this facilitates centralization. Exercises will be progressed at the treating physical therapist's discretion, guided by the principles of maximizing centralization of symptoms and increasing extension range of motion. Not all exercises will be used for every subject, only those which promote centralization or extension range of motion. Exercise progression will be accomplished by increasing the exercise time (up to 5 minutes for sustained exercises) or repetitions (up to 30 repetitions per session), increasing the range of motion towards greater lumbar extension, and adding over-pressure once the end-range of extension range of motion is obtained. Subjects will be instructed to perform all assigned exercise activities at home, every 4-5 hours throughout the day, on days when they do not have a treatment session. Subjects will be provided a copy of an exercise instruction booklet with detailed written descriptions and pictures of the proper performance, frequency, and progression of each exercise. Subject compliance with their home exercise program will be recorded by the treating physical therapist at each treatment session.

The second component of the EOTA will be subject education. Subjects will be educated to maintain the natural lordosis of the lumbar spine while sitting, and will be instructed to avoid prolonged sitting for greater than 20-
30 minutes whenever possible. Additionally, subjects will be educated on the principles of symptom centralization/ peripheralization, and will be given general instructions to discontinue any activities and avoid positions that cause symptoms to peripheralize, and to encourage activities or spinal positions that centralize or improve symptoms.

The third component of the EOTA will be mobilization of the lumbar spine to promote lumbar extension. The mobilization component will consist of a series of up to 20 graded oscillatory mobilizations performed with the subject prone. The physical therapist will apply a posterior-to-anterior mobilization force directed over the spinous process, or laterally over the transverse process, using a grade I - IV mobilization force as described by Maitland [42]. The therapist will select the specific grade(s) and spinal level(s) for mobilization during each treatment session based on the consideration of promoting centralization and lumbar extension range of motion. EOTA + Traction Group

Subjects in the EOTA + traction group will include the EOTA components described above. Mechanical lumbar traction will be applied using a 3D ActiveTrac traction table (Chattanooga Group, Hixson, TN), a motorized, split-table, traction device with a surface that can be adjusted in 3 dimensions (flexion/extension, rotation, and side bending). Subjects will be positioned prone with the table in a neutral position in all planes. If this position 
causes peripheralization or an intolerable increase in symptoms, the table will be adjusted using flexion or side-bending to promote centralization of symptoms and subject comfort as needed.

Static traction will be applied for a maximum of 12 minutes total, which consists of 10 minutes at the desired intensity, plus 1-minute ramp-up and ramp-down times. The intensity of the pull will be $40-60 \%$ of the subject's body weight, adjusted based on the subject's tolerance and symptom response. After 3 minutes of traction, if the table was initially positioned in flexion or side bending, the table will be repositioned as tolerated to achieve a neutral lumbar posture. "Tolerance" will be defined as the ability to achieve a neutral posture (or as close as possible) without peripheralization of symptoms or significant increase in LBP. If the table was initially positioned using both flexion and side-bending, the table will be repositioned first to correct the side-bending accommodation, and then to correct the flexion accommodation. It may not be possible to achieve the goal of a neutral posture during each session, but the goal will be to be as close to neutral posture as tolerated by the subject each session.

At the end of traction treatment, the subject will lie still for 2 minutes prone. For subjects who end their traction treatment in a flat (neutral) position, additional extension positioning will be attempted as follows: the table will be positioned in up to 10 (of extension as tolerated, and the patients will lie still in this position for an additional 2 minutes. For patients who did not end the traction treatment in a flat (neutral) position, no extension positioning will be attempted. At the conclusion of the traction treatment, the subject will be assisted to stand and walk. Flexion, side bending, and sitting positions are to be minimized as much as possible following the traction treatment. If tolerated, the subject will be instructed to perform the same home program of extension-oriented exercises as described in the EOTA group. The mobilization component of the EOTA will be added in the $3^{\text {rd }}$ week for patients in the EOTA plus traction group.

\section{Treatment Side Effects}

A questionnaire for reporting any side effects the subject perceives to be related to treatment procedures will be completed by the subject at the 6-week follow-up. The questionnaire is based on a questionnaire used previously to examine subjects with LBP receiving spinal manipulation [43]. Subjects are asked if they experienced any commonly-reported side effects following treatment including stiffness, muscle spasm, fatigue, or increased radiating symptoms. Subjects will also be able to report side effects not specifically named. For each side effect reported, the subject is asked to report the time of onset relative to the precipitating treatment session $(\leq 24$ hours or $>24$ hours), the duration of the side effect symptoms ( $\leq 24$ hours or $>24$ hours), and severity of symptoms (categorized from 1 "light" to 4 "severe").

\section{Physical Therapist Training and Compliance}

All physical therapists will be trained by the study investigators in the treatment protocols used in this study. One training session of approximately 90 minutes will be used to review all treatment procedures. Each of the participating physical therapy clinics will be equipped with the 3D Active Trac traction table prior to initiation of the study, and physical therapists will have experience with this equipment. Each participating physical therapist will also have prior experience using an EOTA intervention. Participating physical therapists will be trained to provide treatment for subjects in either group in this study. The assignment of a study subject to a participating physical therapist will be done on a pragmatic basis (location of clinic, availability of appointment, etc.) A study treatment folder will be provided to the treating physical therapist each time he or she is assigned a new study subject. The study treatment folder will include a written protocol outline specific to the subject's group assignment, and treatment forms for each physical therapy session to allow the physical therapist to record the specific activities performed during the session and the exercises assigned to the subject for home performance. In addition, each treatment session form will require the physical therapist to record the subject's reported home exercise compliance since the last treatment session. The physical therapist's compliance with the treatment protocols will be recorded and reviewed periodically. The use of any off-protocol co-interventions will be recorded. The subject's compliance with performing the assigned home exercises will be recorded from the physical therapist's treatment forms.

\section{Data Integrity}

The integrity of the data collected throughout the trial will be monitored by regular review of the data collection forms for missing responses, errors, or out of range values.

\section{Data Analysis}

Intention-to-treat principles will be applied to all analyses, with all subjects analyzed with the group to which they were randomized regardless of compliance with treatment. Hypotheses 1 and 2 relate to determining the overall effectiveness of adding traction to an EOTA intervention. Linear mixed models with repeated measures will be used to analyze overall treatment effectiveness. The treating physical therapist and physical therapy clinic will be modeled as random effects. Covariates, treatment 
group, and treatment group by time interaction will modeled as fixed effects. The treatment group by time interaction effect will be used to examine hypotheses 1 and 2 related to the effectiveness of adding traction to an EOTA intervention. Treatment effect sizes with $95 \%$ confidence intervals will be calculated for each follow-up time point. The third hypothesis, examining effectiveness of adding traction to an EOTA intervention based on subjects' status on the preliminary sub-grouping criteria, will be examined with similar procedures with the inclusion of a 3 -way, time by group by sub-grouping status interaction term. Statistical significance will be based on an alpha level of 0.05 for all analyses.

\section{Sample Size and Power}

The sample size calculation is based on examining the differences between treatment groups after 6 weeks on the primary outcome measure (OSW), while preserving adequate power for detecting the sub-grouping effect in hypothesis 3. In our preliminary study [29] we found a treatment effect following the traction intervention of 0.47 , based on a mean between group difference of 6.4 points on the OSW with a sd of 13.7, and a correlation between baseline and follow-up OSW scores of $r=0.57$. We believe our modification of the traction protocol in the present may improve the treatment effect. A sample size of 128 subjects would provide $80 \%$ to detect a slightly larger effect size (0.50). Adjustment for the baseline OSW score will improve power versus an unadjusted comparison of means [44]. Presuming a correlation between baseline and post-treatment OSW scores of 0.57, a sample size of 86 subjects would provide $80 \%$ power for the same treatment effect [44]. To preserve adequate power to examine the interaction effect related to the sub-grouping status in hypothesis 3 , we inflated the sample size based on the size of the anticipated interaction effect relative to the overall treatment effect [45]. In our preliminary study, the magnitude the interaction effect was $1.07 \mathrm{sd}$ between subjects receiving traction who were $\mathrm{SG}+$ compared with subjects receiving EOTA only who were SG+, and was $0.71 \mathrm{sd}$ between subjects receiving traction who were SG+ and those receiving traction who were SG-. If the sub-group effect size is presumed to be at least 1.0, no further sample size inflation would be required to maintain the same power as for the overall treatment effect, while a sub-group effect size of 0.71 would require an inflation of approximately 1.5 [45]. Inflating the sample size by 1.2 results in a sample size of 104 subjects. Recruiting 120 subjects (60 per group) allows for a dropout rate of approximately $13 \%$. Our sample size estimate would be under-powered if a large clustering effect due to subjects within clinical sites were present [46]. We are presuming that our use of standardized protocols will make any clustering effects negligible. If this presumption proves incorrect the study could be under-powered. Conversely, our sample size calculation accounts for adjustment for baseline OSW values. We will adjust for additional baseline co-variates which may increase power. A sample size of 120 subjects provides $80 \%$ power to detect a between-group difference in low back or leg pain rating of 1.0 points presuming a sd of 2.0 , and a difference in the percentage of successful patients of $25 \%$ based on dichotomization of the global rating of change scale.

\section{Discussion}

This study will examine the effectiveness of adding a standardized protocol of lumbar mechanical traction to an extension-oriented treatment approach for individuals with LBP and nerve root irritation who are receiving nonsurgical care. The study will further examine if the addition of the traction protocol is particularly beneficial in a sub-group of subjects defined by the presence of clinical examination factors identified in preliminary research. The study will examine the overall effect of the treatment received, and the interaction between treatment received and the sub-group status. This is the recommended strategy for determining if treatment effects differ across subgroups [47]. The sample size has been adjusted to preserve adequate power for the analysis of the sub-grouping interaction effect [45]. There is currently a lack of evidence to inform clinical decision-making for the non-surgical management of LBP and nerve root irritation, even though a period of non-surgical management is recommended for most patients. The overall goal of this study is to provide evidence that can improve decision-making for these patients.

\section{Competing interests}

We acknowledge a potential competing interest in the funding of this study, which is provided by DJO incorporated, Vista, California, USA. DJO is the parent company of Chattanooga, Inc., the manufacturer of the 3D ActiveTrac traction table used in this study.

\section{Authors' contributions}

JF and JC conceived of this study and participated in its design. AT and GB contributed to the design of this study. AT will serve as the study coordinator. All authors have read and approved the final manuscript.

\section{Author Details}

'Rehabilitation Agency, Intermountain Healthcare, Salt Lake City, Utah, USA, 2Department of Physical Therapy, The University of Utah, Salt Lake City, Utah, USA and ${ }^{3}$ U.S. Army-Baylor University, Fort Sam Houston, Texas, USA

Received: 10 December 2009 Accepted: 30 April 2010

Published: 30 April 2010

\section{References}

1. Deyo RA, Mirza SK, Martin BI: Back pain prevalence and visit rates: estimates from U.S. national surveys, 2002. Spine 2006, 31:2724-2727.

2. Abenhaim L, Rossignol M, Gobeille D, Bonvalot $Y$, Fines $P$, Scott $S$ : The prognostic consequences in the making of the initial medical diagnosis of work-related back injuries. Spine 1995, 20:791-795. 
3. Tubach F, Beaute J, Leclerc A: Natural history and prognostic indicators of sciatica. J Clin Epidemiol 2004, 57:174-179.

4. Stafford MA, Peng P, Hill DA: Sciatica: a review of history, epidemiology, pathogenesis and the role of epidural steroid injection in management. Br J Anaesth 2007, 99:461-473.

5. Konstantinou K, Dunn KM: Sciatica: review of epidemiological studies and prevalence estimates. Spine 2008, 33:2464-2472.

6. Balague F, Nordin M, Sheikhzadeh A, Echegoyen AC, Brisby H, Hoogewoud HM, Fredman P, Skovron ML: Recovery of severe sciatica. Spine 1999, 24:2516-2524

7. Selim AJ, Ren XS, Fincke G, Deyo RA, Rogers W, Miller D, Linzer M, Kazis L: The importance of radiating leg pain in assessing health outcomes among patients with low back pain. Results from the Veteran Health Study. Spine 1998, 23:470-474.

8. Engel CC, von Korff M, Katon WJ: Back pain in primary care: predictors of high health-care costs. Pain 1996, 65:197-204.

9. Peul WC, Hout WB van den, Brand R, Thomeer RT, Koes BW: Prolonged conservative care versus early surgery in patients with sciatica caused by lumbar disc herniation: two year results of a randomised controlled trial. BMJ 2008, 336:1355-1358.

10. Andersson GB, Brown MD, Dvorak J, Herzog RJ, Kambin P, Malter A, McCulloch JA, Saal JA, Spratt KF, Weinstein JN: Consensus summary of the diagnosis and treatment of lumbar disc herniation. Spine 1996, 21(suppl):s75-s78

11. Atlas SJ, Keller RB, Wu YA, Deyo RA, Singer DE: Long-term outcomes of surgical and nonsurgical management of sciatica secondary to a lumbar disc herniation: 10 year results from the Maine lumbar spine study. Spine 2005, 30:927-934

12. Cummins J, Lurie JD, Tosteson TD, Hanscom B, Abdu WA, Birkmeyer NJ, Herkowitz H, Weinstein J: Descriptive epidemiology and prior healthcare utilization of patients in the Spine Patient Outcomes Research Trial's (SPORT) three observational cohorts: disc herniation spinal stenosis and degenerative spondylolisthesis. Spine 2006, 31:806-814

13. Luijsterburg PA, Verhagen AP, Ostelo RW, Hoogen HJ van den, Peul WC, Avezaat CJ, Koes BW: Physical therapy plus general practitioners' care versus general practitioners' care alone for sciatica: a randomised clinical trial with a 12-month follow-up. Eur Spine J 2008, 17:509-517.

14. Luijsterburg PA, Lamers LM, Verhagen AP, Ostelo RW, Hoogen HJ van den, Peul WC, Avezaat CJ, Koes BW: Cost-effectiveness of physical therapy and general practitioner care for sciatica. Spine 2007, 32:1942-1948.

15. Jewell DV, Riddle DL: Interventions that increase or decrease the likelihood of a meaningful improvement in physical health in patients with sciatica. Phys Ther 2005, 85:1139-1150.

16. Luijsterburg PA, Verhagen AP, Ostelo RW, van Os TA, Peul WC, Koes BW: Effectiveness of conservative treatments for the lumbosacral radicular syndrome: a systematic review. Eur Spine J 2007, 16:881-899.

17. Vroomen PCAJ, de Krom MC, Slofstra PD, Knottnerus JA: Conservative treatment of sciatica: a systematic review. J Spinal Dis 2000, 13:463-469

18. Crisp EJ, Cyriax JH, Christie BG: Discussion on the treatment of backache by traction. Proc R Soc Med 1955, 48:805-814.

19. Chou R, Huffman LH: Nonpharmacologic therapies for acute and chronic low back pain: a review of the evidence for an American Pain Society/American College of Physicians clinical practice guideline. Ann Intern Med 2007, 147:492-504.

20. Clarke J, van Tulder M, Blomberg S, de Vet $\mathrm{H}$, Heijden G van der, Bronfort G: Traction for low back pain with or without sciatica: an updated systematic review within the framework of the Cochrane collaboration. Spine 2006, 31:1591-1599.

21. van Tulder MW, Koes BW, Seitsalo S, Malmivaara A: Outcome of invasive treatment modalities on back pain and sciatica: an evidence-based review. Eur Spine J 2006, 15(suppl):S82-S92.

22. Foster NE, Thompson KA, Baxter GD, Allen JM: Management of nonspecific low back pain by physiotherapists in Britain and Ireland. A descriptive questionnaire of current clinical practice. Spine 1999, 24:1332-1342

23. Gracey JH, McDonough SM, Baxter GD: Physiotherapy management of low back pain. Spine 2002, 27:406-411.

24. Mikhail C, Korner-Bitensky N, Rossignoi M, Dumas JP: Physical therapists' use of interventions with high evidence of effectiveness in the management of a hypothetical typical patient with acute low back pain. Phys Ther 2005, 85:1151-1167.

25. Poitras S, Blais R, Swaine B, Rossignol M: Management of work-related low back pain: a population-based survey of physical therapists. Phys Ther 2005, 85:1 168-1181.

26. Gay RE, Brault JS: Evidence-based management of chronic low back pain with traction therapy. Spine J 2008, 8:234-242.

27. Krause M, Refshauge KM, Dessen M, Boland R: Lumbar spine traction: evaluation of effects and recommended application for treatment. Man Ther 2000, 5:72-81.

28. Harte AA, Gracey JH, Baxter GD: Current use of lumbar traction in the management of low back pain: results of a survey of physiotherapists in the United Kingdom. Arch Phys Med Rehabil 2005, 86:1 164-1169.

29. Fritz JM, Lindsay W, Matheson JW, Brennan GP, Hunter SJ, Moffitt SD, Swalberg A, Rodriquez B: Is there a subgroup of patients with low back pain likely to benefit from mechanical traction?: Results of a randomized clinical trial and subgrouping analysis. Spine 2007, 32:E793-E800

30. Saunders HD, Ryan RS: Spinal traction. In Evaluation Treatment and Prevention of Musculoskeletal Disorders Volume 1. Chaska, MN: The Saunders Group, Inc:; 2004:301-325

31. Fritz JM, Irrgang JJ: A comparison of a modified Oswestry disability questionnaire and the Quebec back pain disability scale. Phys Ther 2001, 81:776-788.

32. Hancock MJ, Herbert RD, Maher CG: A guide to interpretation of studies investgating subgroups of responders to physical therapy interventions. Phys Ther 2009, 89:698-704.

33. Laupacis A, Sekar N, Stiell IG: Clinical prediction rules: a review and suggested modifications of methodological standards. JAMA 1997, 277(6):488-494

34. Waddell G, Newton M, Henderson I, Somerville D, Main CJ: A FearAvoidance Beliefs Questionnaire ( $F A B Q$ ) and the role of fear-avoidance beliefs in chronic low back pain and disability. Pain 1993, 52:157-168.

35. Sullivan MJ, Bishop SR, Pivik J: The pain catastrophizing scale: development and validation. Psychol Assess 1995, 7:524-532.

36. Bombardier C: Outcome assessment in the evaluation of treatment of spinal disorders: summary and general recommendations. Spine 2000, 25(24):3100-3103.

37. Frost H, Lamb SE, Stewart-Brown S: Responsiveness of a patient specific outcome measure compared With the Oswestry disability index v2.1 and Roland and Morris disability questionnaire for patients with subacute and chronic low back pain. Spine 2008, 33:2450-2457.

38. Walsh TL, Hanscom B, Lurie JD, Weinstein JN: Is a condition-specific instrument for patients with low back pain/leg symptoms really necessary? The responsiveness of the Oswestry Disability Index MODEMS and the SF-36. Spine 2003, 28:607-615.

39. Childs JD, Piva SR, Fritz JM: Responsiveness of the numeric pain rating scale in patients with low back pain. Spine 2005, 30:1331-1335.

40. Jaeschke R, Singer J, Guyatt GH: Measurement of health status: ascertaining the minimal clinically important difference. Control Clin Trials 1989, 10:407-415.

41. EuroQol Group: EuroQol-A new facility for the measurement of healthrelated quality of life. Health Policy 1990, 16:199-208.

42. Maitland GD, Hengeveld E, Banks K: Vertebral Manipulation. 6th edition. Oxford: Butterworth Heinemann; 2000

43. Caigne B, Vinck E, Beernaert A, et al:: How common are side effects of spinal manipulation and can these side effects be predicted? Man Ther 2004, 9:151-156.

44. Borm GF, Fransen J, Lemmens WAJG: A simple sample size formula for analysis of covariance in randomized clinical trials. J Clin Epidemiol 2007, 60:1234-1238.

45. Brookes ST, Whitely E, Egger M, Smith GD, Mulheran PA, Peters TJ: Subgroup analyses in randomised trials: risks of subgroup specific analyses; power and sample size for the interaction test. J Clin Epidemiol 2004, 57:229-236.

46. Lee KJ, Thompson SG: Clustering by health professional in individually randomised trials. BM/ 2005, 330:142-144

47. Oxman AD, Guyatt GH: A consumer's guide to subgroup analyses. Ann Intern Med 1992, 116:78-84. 
48. Fritz JM, Delitto A, Vignovic M, Busse RG: Inter-rater reliability of judgments of the centralization phenomenon and status change during movement testing in patients with low back pain. Arch Phys Med Rehabil 2000, 81:57-61.

49. Hudgins WR: The crossed straight leg raising test: a diagnostic sign of herniated disc. J Occup Med 1979, 21:407-408.

\section{Pre-publication history}

The pre-publication history for this paper can be accessed here: http://www.biomedcentral.com/1471-2474/11/81/prepub

\section{doi: $10.1186 / 1471-2474-11-81$}

Cite this article as: Fritz et al., A randomized clinical trial of the effectiveness of mechanical traction for sub-groups of patients with low back pain: study methods and rationale BMC Musculoskeletal Disorders 2010, 11:81

Submit your next manuscript to BioMed Central and take full advantage of:

- Convenient online submission

- Thorough peer review

- No space constraints or color figure charges

- Immediate publication on acceptance

- Inclusion in PubMed, CAS, Scopus and Google Scholar

- Research which is freely available for redistribution

Submit your manuscript at www.biomedcentral.com/submit
() BioMed Central 